\title{
The digital pill
}

\section{Tracking medication adherence through electronic modalities}

\author{
Steven Wong, Victoria Chan \\ Faculty Reviewer: Rajendra Harricharan, MBBS, FRCPC (Department of Psychiatry)
}

\section{ABSTRACT}

Compliance with prescription medication regimens is poor in patients who suffer from chronic conditions as well as from diseases that affect public health. It is thought that improving medication adherence can have a profound effect on patient health, though medication compliance remains a problem despite the availability of many modalities. Recently, digitalizing medication adherence was made possible by Proteus Digital Health, Inc using an ingestible sensor that emits an electric field upon digestion. This signal is detected by an externally worn adhesive monitor, which records the time at which the signal is received, along with other biometric markers such as heart rate. This system is currently under United States Food and Drug Administration (FDA) review for use in a combination pill that also contains aripiprazole, a partial dopamine agonist used for the treatment of certain serious psychiatric conditions. Digitalizing medication adherence can have tremendous applications in all fields of medicine, though issues of drug costs, patient privacy, and patient autonomy may need to be addressed.

\section{INTRODUCTION}

Lack of adherence to prescription oral medication is an alarming problem in our healthcare system. Studies in the past decade have shown that approximately $50 \%$ of patients with chronic illness do not take their medications as prescribed. ${ }^{1}$ Being forgetful is the commonest reason for poor adherence, though some patients may voluntarily choose not to take their medications, perceiving medication as unnecessary, or being fearful of any associated side effects. $^{2}$ The latter is a cause for concern for public health, particularly with communicable diseases such as tuberculosis that can be effectively managed through medication.,4 Poor compliance to prescription medication regimens puts a tremendous burden on the medical system, as noncompliant patients are more likely to be readmitted to the hospital or clinic, and possibly be prescribed more and/or more potent medication that they may not otherwise need. This ultimately leads to poorer health outcomes, and it has been argued that increasing the effectiveness of adherence interventions may have a far greater impact on patient health than any improvement in specific medical treatments. ${ }^{5}$ This problem therefore compels the need to identify modalities to accurately ensure medication compliance in at-risk patients.

\section{CURRENT MODALITIES TO IMPROVE MEDICAL ADHERANCE}

The issue of medication nonadherence has been tackled on various fronts to varying degrees of success. Physicians and pharmacists can play an essential role by simplifying regimen characteristics, making the regimen compatible with the patient's lifestyle and daily schedule, and educating patients about their medications and why they are taking them. ${ }^{1,6}$ Additionally, a variety of adherence aids are available to patients including pill organizers, blister packs, and reminders/alarms. ${ }^{7,8}$ For instance, electronic reminders in the form of pagers, cellphones, and text messages have been shown to improve medication refill adherence in type 2 diabetes mellitus patients. ${ }^{9}$ Another approach to improving medication compliance is directly observed therapy (DOT), which arose from the need for strict medication compliance in managing tuberculosis to limit the spread of the infectious agent. ${ }^{10}$ DOT involves a healthcare worker or designated individual watching the patient swallow all doses of their medications. DOT is currently the World Health Organization standard for ensuring medication compliance for tuberculosis. Although this approach should theoretically be entirely effective in ensuring tuberculosis medication compliance, the literature suggests that DOT is no better than standard approaches in many communities globally. ${ }^{10}$ These results may reflect the fact that DOT is a time- as well as resource-intensive procedure that requires efficient and properly trained staff, which is unavailable in many communities. ${ }^{10}$ Furthermore, the process of being regularly monitored can be demeaning to some patients, who then may be reluctant to complete their medication regimen..$^{10}$ Despite a plethora of modalities aimed to improve medication adherence, not one of them has demonstrated complete medication compliance. An ideal modality for ensuring medication compliance would be one that not only is highly accurate in detecting if the medication has been taken, but also does not require many resources.

\section{DIGITALIZING MEDICAL ADHERENCE}

Monitoring medication adherence through electronic means was recently made possible through the works of Proteus Digital Health, Inc This FDA-approved modality uses an ingestible sensor the size of a grain of sand that is incorporated into a pill. Upon entry into the stomach, the electrolytes found on the sensor's surface (copper and magnesium) react with the electrolytes found in gastric acid to form a biogalvanic-like battery. ${ }^{11}$ The sensor utilizes this current to generate an electric field, which is detected by an externally worn adhesive monitor called the Proteus Personal Monitor. ${ }^{11}$ The ingestible sensor is capable of communicating with the mon- 
itor for 5 to 10 minutes before the sensor subsequently becomes inactivated, passes through the rest of the gastrointestinal tract, and is excreted together with feces. Along with recording when the ingestible sensor signal is detected, the monitor is also capable of recording other parameters such as heart rate and physical activity, making it a helpful tool for both clinicians and researchers. ${ }^{11}$ Data from the monitor are encrypted and transmitted to the patient's mobile device via Bluetooth technology, and are subsequently uploaded to a central server, making it possible to integrate data collected by the monitor into electronic medical records. ${ }^{11}$ This system has demonstrated high accuracy in detecting the ingestible tracer, low adverse effects in patients (limited to skin rash and 1 case of nausea), and a high rate of patient acceptance. ${ }^{12}$ It also requires minimal training, making it an optimal candidate for monitoring medication adherence.

In September 2015, Proteus Digital Health, Inc filed a joint FDA application with Otsuka America Pharmaceutical, Inc for approval of a combination pill that included both an ingestible sensor and aripiprazole (trade name Abilify). Aripiprazole is a partial dopamine agonist that has been shown to be effective in the management of psychiatric diseases including schizophrenia, bipolar disease, and depression. This application, the first of its kind, hopes to demonstrate the potential of digitalizing medication adherence for patients experiencing psychiatric illnesses, where noncompliance rates to medication therapy can be high. Moreover, the outcome of this FDA application, if successful, will undoubtedly influence other fields of medicine. Though not currently approved for clinical use, Belknap et al utilized an earlier prototype of the system designed by Proteus Digital Health, Inc to demonstrate markedly improved medication compliance for the management of tuberculosis disease. ${ }^{12}$ Digitalizing medication compliance can also have a tremendous impact in the field of cardiovascular diseases, where nonadherence to medications has been shown in $60 \%$ of patients, as well as in a variety of other chronic conditions including rheumatoid arthritis, diabetes mellitus, and sickle cell anemia. ${ }^{13-15}$

Despite the potential to revolutionize healthcare, there are several important limitations and concerns to consider with digitalizing medication adherence. The design of this particular ingestible sensor limits its application to drugs taken orally at the present time. Secondly, there are concerns that incorporating an ingestible sensor into current medication will significantly increase drug costs. As medication affordability is another barrier with medication adherence, particularly for patients without drug insurance coverage, this may make this medication adherence strategy unaffordable for the patients who need it the most. Patient privacy is another concern with digitalizing medical adherence, as there are risks with recording and storing personal health information onto a central server not owned by the patient. However, Proteus Digital Health, Inc has advertised its product as utilizing industry-standard encryption techniques to allow for the secure collection and storage of personal health information. ${ }^{11}$ Similar to patient privacy is the issue that some patients may view this strategy of monitoring medication compliance as unnecessary, excessive, and an act of healthcare provider paternalism. These feelings will need to be addressed by the healthcare provider prior to initiation of thera- py. Lastly, while this technology effectively monitors medication adherence, it must be supplemented by appropriate and effective patient counseling in order for it to be truly effective in improving patient compliance to medication.

\section{SUMMARY}

Digitalizing medication adherence is a very promising modality for improving medication compliance. Compared to other existing modalities, the ingestible sensor accurately records the time at which patients ingest their medications, providing healthcare providers with longitudinal data that they can use to effectively counsel patients about medication compliance. Currently, an FDA application has been filed for use of this technology with aripiprazole, though it would not be surprising if the ingestible sensor were to be incorporated into other medications in the future. Despite the benefits, issues of drug costs, patient privacy, and patient autonomy may need to be addressed before it can be widely used in a clinical setting. However, the "digital pill" offers an innovative solution to a longstanding problem in healthcare, and may be an essential tool in the future physician's toolbox in improving patient health outcomes.

\section{REFERENCES}

1. Brown MT, Bussell JK. Medication adherence: WHO cares? Mayo Clin Proc. 2011 Apr;86(4):304-14.

2. Ho PM, Bryson CL, Rumsfeld JS. Medication adherence: its importance in cardiovascular outcomes. Circulation. 2009 Jun 16;119(23):3028-35.

3. Munro SA, Lewin SA, Smith HJ, Engel ME, Fretheim A, Volmink J. Patient adherence to tuberculosis treatment: a systematic review of qualitative research. PLoS Med. 2007 Jul 24;4(7):e238.

4. Tang Y, Zhao M, Wang Y, Gong Y, Yin X, Zhao A, et al. Non-adherence to anti-tuberculosis treatment among internal migrants with pulmonary tuberculosis in Shenzhen, China: a cross-sectional study. BMC Public Health. 2015;15:474.

5. Sabaté E. Adherence to long-term therapies: evidence for action. WHO. 2003;1-194.

6. Jimmy B, Jose J. Patient medication adherence: measures in daily practice. Oman Med J. 2011 May;26(3):155-9.

7. Huang HY, Maguire MG, Miller ER, Appel LJ. Impact of pill organizers and blister packs on adherence to pill taking in two vitamin supplementation trials. Am J Epidemiol. 2000 Oct 15;152(8):780-7.

8. Petersen ML, Wang Y, van der Laan MJ, Guzman D, Riley E, Bangsberg DR. Pillbox organizers are associated with improved adherence to HIV antiretroviral therapy and viral suppression: a marginal structural model analysis. Clin Infect Dis. 2007 Oct 1;45(7):908-15.

9. Vervloet M, van Dijk L, Santen-Reestman J, van Vlijmen B, van Wingerden P, Bouvy ML, et al. SMS reminders improve adherence to oral medication in type 2 diabetes patients who are real time electronically monitored. Int J Med Inform. 2012 Sep;81(9):594-604.

10. Karumbi J, Garner P. Directly observed therapy for treating tuberculosis. Cochrane database Syst Rev. 2015;5:CD003343.

11. DiCarlo L, Moon G, Intondi A, Duck R, Frank J, Hafazi H, et al. A digital health solution for using and managing medications: wirelessly observed therapy. IEEE Pulse. 3(5):23-6.

12. Belknap R, Weis S, Brookens A, Au-Yeung KY, Moon G, DiCarlo L, et al. Feasibility of an ingestible sensor-based system for monitoring adherence to tuberculosis therapy. PLoS One. 2013;8(1):e53373. 


\section{MEDICINE AND TECHNOLOGY}

13. Briesacher BA, Andrade SE, Fouayzi H, Chan KA. Comparison of drug adherence rates among patients with seven different medical conditions. Pharmacotherapy. 2008 Apr;28(4):437-43.

14. Baroletti S, Dell'Orfano H. Medication adherence in cardiovascular disease. Circulation. 2010 Mar 30;121(12):1455-8.

15. Creary SE, Gladwin MT, Byrne M, Hildesheim M, Krishnamurti L. A pilot study of electronic directly observed therapy to improve hydroxyurea adherence in pediatric patients with sickle-cell disease. Pediatr Blood Cancer. 2014 Jun;61(6):1068-73. 